\title{
Caching Games between ISP in Information Centric Network
}

\author{
Hamid Garmani ${ }^{1}$, Mohamed Baslam ${ }^{2}$ and Mostafa Jourhmane ${ }^{3}$ \\ ${ }^{1,2,3}$ Information Processing and Decision Support Laboratory, Faculty of Sciences \\ and Technics, Sultan Moulay Slimane University, Beni Mellal, Morocco \\ Igarmani.hamid@gmail.com, ${ }^{2}$ baslam.med@gmail.com,3jourhman@hotmail.com
}

\begin{abstract}
Information Centric Network ICN is a future network architecture, wich has recently emerged to improve performance of the current Internet, in ICN content is located by name not by location. This paper develop game theoretic models to study the interaction between ISP in an ICN model where the ISP is motivated to cache content. We analyze the behavior of ISPs in terms of their strategies within the framework of non-cooperative game theory. In turn, the subscribers' demand for the service of an ISP depends not only on their strategies, but also upon those proposed by all of its competitors. We provide some interesting results regarding the Nash equilibrium of this game. More precisely, we showed existence and uniqueness of the Nash equilibrium under some conditions. Furthermore, we introduce learning algorithms that may lead ISPs to learn their strategies at Nash equilibriums. Extensive simulations show the convergence to the Nash equilibrium and give the effect of caching in ISPs strategies. This paper shows through economic analysis that caching is beneficial for ISP and users.
\end{abstract}

Keywords: Caching, ISP, Game Theory, Nash Equilibrium, ICN

\section{Introduction}

Internet traffic is rapidly increasing, due to the proliferation of social media, such as Facebook, Twitter, and Youtube. This high increase in demand for content on the internet and the need of new approaches that control the large volume of information have motivated the development of new approach called Information Centric Network ICN. This last (ICN) is a new communication paradigm which aims to increase the efficiency of content delivery and content availability. The main idea in ICN: 1) users send requests with the name of the content, without specifying the location of the content 2) Every ICN node can cache and serve the requested content. The advantages motivating the ICN approach Scalable, Persistent, Security, Mobility, etc., [1]. To fulfill that purpose, several architectures have been proposed for ICN: CCN [11], DONA[14], etc.

In spite of these expected technical benefits, broadly investigated by the research community, ICN has so far stayed only in the research literature, contrary to Content Delivery Networks (CDN) or HTTP caches that enjoy a large deployment. This is because, technical benefits are not sufficient to persuade huge providers (like ISPs and CPs) to switch to ICN, if they do not have clear economic incentives. As such, the investigation of the economic implications of ICN may have prominent impact.

Several works have been done to address the problem of the economic implications of caching [13] [20] [6] [8]. So far, however, there has been little discussion about ISP caching with regard to the competition between the ISPs and CPs in ICN. In [13] [20] the authors' investigated ISP caching in a game-theoretic

Received (January 4, 2018), Review Result (March 10, 2018), Accepted (March 14, 2018) 
Our work seeks to address the issue of economic incentives in ISP cache by formulating and analyzing a new pricing and caching model in a competitive context, where all ISP aim to maximize their own profits.

Our contribution in this paper is:

- We present new features in the mathematical modeling that include the price to access to the content in the cache, the number of items in the cache and the ISPs revenues.

- We model the interactions in price, quality of service and caching among ISPs in ICN. The competitive problem between ISPs formulate as a noncooperative game.

- Users' behavior is modeled as a function of ISP strategies (i.e., network access price, price for access to the content in the cache, quality of service QoS and the number of items in the cache).

- We analytically prove the existence and uniqueness of Nash equilibrium in non-cooperative game between ISPs, which means that there exists a stable state where all ISPs do not have an incentive to change their strategies. Therefore, our model provides economic incentives for caching content, and ensures the existence of an equilibrium for keeping the economy stable and achieving economic growth. The best response algorithm is used to find the Nash equilibrium point.

- We complement our analysis with numerical results that shows a positive impact of caching on network access price, quality of service and revenue of ISP. This means that both the ISP and end-users can receive benefit from caching investment.

The rest of this work is organized as follows. In Section 2 related work. In Section 3 we describe the system model and we introduce a new demand and utility functions. In Section 4 we formulate a non-cooperative game, and we proof the existence and uniqueness of Nash equilibrium solution. Then, we present numerical results in Section 5. In Section 6 Conclusion.

\section{Related Work}

The existing literature has studied a number of problems related to caching in the small cell network, base station, cloud radio access networks, ISP and CP. In [12] the authors have presented a survey of caching at ICN. The authors in [22] determine optimally which content objects are placed at which ICN nodes. The authors in [19] introduced proactive caching to reduce congestion at the backhaul link and use D2D communication for sharing local content without multiple base station request. Several approaches such as base station assisted D2D network and other schemes based on caching at the user device, show the improvement of network caching. In [10] the authors have used mean field game to study the problem of distributed caching in dense wireless small cell network (SCN). The aim of this work is to define a caching policy that reduces the load on the capacity limited backhaul links. The authors in [23] have proposed a proactive encoded caching policy based on the mobility information of the users. The proposed caching policy aims to minimize the fraction of file requests that are served by the main base station. In [9] the authors formulate the caching problem as many to many matching game to reduce load on the backhaul link and transmission delay. The authors in [5] [4] studied proactive caching at the baseband units (BBUs) in cloud radio access networks (CRANs). The authors also develop a novel proactive caching framework that can accurately predict and cache user content while minimizing backhaul traffic 
and delay with a CRAN.

In [17] the authors identified that ISP need to be involved in the process of cache deployment due to their ownership of the network. The authors also identify the different business model of ISP in a caching environment and utilize game theory to evaluate these possible business models. The authors in [16] studied a new business model where an ISP charge CP for caching service because CP benefit from more efficient content distribution. A pricing model proposed in [21] to study the economic incentive for caching and sharing content in ICN, where ICN consists of access ICN, transit ICN and CP. Therefore, a unique Nash Equilibrium (NE) exists in a non-cooperative pricing caching game. The result of this work shows the case where caching investment is profitable for access ISP. In [13] the authors considered a non-cooperative game between CP and ISP in ICN, where CP and ISP playing with their pricing strategies and fixing the caching strategies. Depending on the caching cost, the ISP may be incentivized to cache content in ICN. The authors in [8] analyzed the impact of caching cost in joint caching and pricing strategies in ICN with one CP, tow access ICN, one transit ICN. Competition between entities modeled as a non-cooperative game and observes that caching strategy depending on the caching cost and price at the transit ICN. In [20] the authors studied a noncooperative game between one CP and one ISP in ICN, where ISP cache content. It shows that caching investment is beneficial for ISP and CP. The authors in [6] modeled the caching game between $\mathrm{CP}$ and ISP as a cooperative game, where ISP cache a fraction of content. Then, CP and ISP share both the cost of caching and profit of caching. Also, a competition between CPs modeled as a non-cooperative game to capture the negative impact on demand when the ISP cache content of other CPs. In contrast, in this paper, we formulate the interaction of caching, QoS and pricing in a non-cooperative game between ISPs in ICN, where the ISPs can adjust their caching, QoS and pricing strategies. Our results provide insights on the provision of incentives for caching in the context of ISP, a key requirement for a successful implementation of the ICN architecture in the future Internet.

\section{Problem Modeling}

We consider a simplified networking market with one CP, M ISP and an arbitrary number of users who can switch from one ISP to another. All end users can access the contents of the $\mathrm{CP}$ only through the network infrastructure provided by the ISP while $\mathrm{CP}$ provides the content for the users. Figure 1 shows the monetary flow among different entities with various prices described in table 1 . The network economy depends on three effective factor pricing, caching and quality of service QoS. Under the assumption that each ISP can have access to all content, it can decide to either cache the entire or a portion of the requested content. Let $\mathrm{N}$, the number of items that the CP sells. The caching strategy adopted by each ISP is denoted by $K$ that take values in the interval $[0, N]$. Each access ISP sets two different prices: (1) the network price per unit data for transporting the content to end users; and (2) the price per unit data for providing content from its cache. Each ISP allocates a Bandwidth B and advertises to users a quality of service QoS.

To model the behavior of users, we have considered content demand at each ISP to be a linear function of the strategies of all ISPs. 


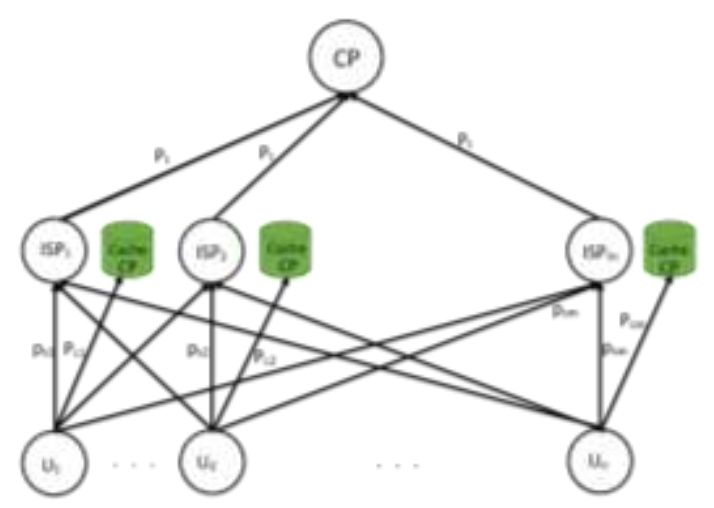

Figure 1. ICN Architecture

Table 1. Summary of Notation

\begin{tabular}{|c|l|}
\hline Notation & Description \\
\hline $\mathrm{N}$ & Number of items that the CP sells \\
\hline $\mathrm{M}$ & Number of ISP \\
\hline$p_{s_{j}}$ & Network access price of $I S P_{j}$ \\
\hline$p_{c_{j}}$ & Price to access to the content in the cache of $I S P_{j}$ \\
\hline$q_{s_{j}}$ & Quality of service of $I S P_{j}$ \\
\hline$k_{j}$ & Number of cached items of $I S P_{j}$ \\
\hline$c_{j}$ & Caching cost of $I S P_{j}$ \\
\hline$v_{j}$ & Unit backhaul bandwidth cost \\
\hline$d_{j}$ & The total potential demand of users for $I S P_{j}$ \\
\hline$\alpha_{j}^{m}$ & Sensitivity of $I S P_{j}$ to price $p_{s_{m}}$ of $I S P_{m}$ \\
\hline$\beta_{j}^{m}$ & Sensitivity of $I S P_{j}$ to QoS $q_{s_{m}}$ of $I S P_{m}$ \\
\hline$\sigma_{j}^{m}$ & Sensitivity of $I S P_{j}$ to price $p_{c_{m}}$ of $I S P_{m}$ \\
\hline$\gamma_{j}^{m}$ & Sensitivity of $I S P_{j}$ to number of cached items $k_{m}$ of $I S P_{m}$ \\
\hline$D_{j}$ & Demand of $I S P_{j}$ \\
\hline$B_{j}$ & Backhaul bandwidth needed to serve the demand $D_{j}$ \\
\hline
\end{tabular}

\subsection{Demand Model}

The demand of $I S P_{j}$ is linear function with respect to the network access price $p_{s_{j}}$, price to access to the content in cache $p_{c_{j}}$, quality of service QoS $q_{s_{j}}$ and number of cached items $k_{j}$, see [3]. This demand depend also on the price $\mathbf{p}_{s_{-j}}$, price $\mathbf{p}_{c_{-j}}$, quality of service QoS $\mathbf{q}_{s_{-j}}$ and number of cached items $\mathbf{k}_{-j}$ where $\mathbf{p}_{s_{-j}}=\left(p_{s_{1}}, p_{s_{2}}, \ldots, p_{s_{i-1}}, p_{s_{i+1}}, \ldots, p_{s_{M}}\right)$. Then the demand function depends on $\mathbf{p}_{s}=\left(p_{s_{1}}, \ldots, p_{s_{M}}\right), p_{c}=\left(p_{c_{1}}, \ldots, p_{c_{M}}\right), \quad \mathbf{q}_{s}=\left(p_{q_{1}}, \ldots, p_{q_{M}}\right) \quad$ and $\mathbf{k}=\left(k_{1}, \ldots, k_{M}\right)$. 
$D_{j}$ decreasing w.r.t $p_{s_{j}}, p_{c_{j}}$ and increasing w.r.t $p_{s_{m}}, p_{c_{m}}, j \neq m . D_{j}$ increasing w.r.t $q_{s_{j}}, k_{j}$ and decreasing w.r.t $q_{s_{m}}, k_{m}, j \neq m$. The demand function can be write as follows:

$$
\begin{aligned}
& D_{j}\left(\mathbf{p}_{s}, \mathbf{p}_{c}, \mathbf{q}_{s}, \mathbf{k}\right)=d_{j}-\alpha_{j}^{j} p_{s_{j}}+\beta_{j}^{j} q_{s_{j}}+\sigma_{j}^{j} k_{j}-\gamma_{j}^{j} p_{c_{j}} \\
& +\sum_{m=1, m \neq j}^{M}\left(\alpha_{j}^{m} p_{s_{m}}-\beta_{j}^{m} q_{s_{m}}-\sigma_{j}^{m} k_{m}+\gamma_{j}^{m} p_{c_{m}}\right)
\end{aligned}
$$

The parameter $d_{j}$ expresses the total potential demand of users. $\alpha_{j}^{m}, \beta_{j}^{m}, \gamma_{j}^{m}$ and $\sigma_{j}^{m}$ are positive constant represent respectively the sensitivity the demand function the $I S P_{j}$ to price $p_{s_{m}}$, QoS $q_{s_{m}}$, price $p_{c_{m}}$ and number of cached items $k_{m}$.

\section{Assumption 1}

$$
\sum_{m=1}^{M} \alpha_{j}^{m}=1, \quad \sum_{m=1}^{M} \beta_{j}^{m}=1, \quad \sum_{m=1}^{M} \gamma_{j}^{m}=1, \quad \sum_{m=1}^{M} \sigma_{j}^{m}=1, i=1, \ldots, M .
$$

The sensitivity $\alpha$ satisfy:

$$
\alpha_{j}^{j} \geq \sum_{m, m \neq j} \alpha_{j}^{m}
$$

The sensitivity $\beta$ satisfy:

$$
\beta_{j}^{j} \geq \sum_{m, m \neq j} \beta_{j}^{m}
$$

The sensitivity $\sigma$ satisfy:

$$
\sigma_{j}^{j} \geq \sum_{m, m \neq j} \sigma_{j}^{m}
$$

The sensitivity $\gamma$ satisfy:

$$
\gamma_{j}^{j} \geq \sum_{m, m \neq j} \gamma_{j}^{m}
$$

Assumption 1 will be needed to ensure the uniqueness of the resulting equilibrium. The assumption 1 means that the influence of price (resp Qos and number of cached items) the ISP on its demand is greater than the influence of the prices (resp Qos and number of cached items) of its opponents on its demand.

\subsection{Effect of Caching}

When $I S P_{j}$ cache k items:

- The new demand of CP through $I S P_{j}$ is $\left(N-k_{j}\right) D_{j}$.

- $B_{j}$ Backhaul bandwidth needed to serve the demand $D_{j},\left(N-k_{j}\right) B_{j}$ the new Backhaul bandwidth needed to serve the demand $\left(N-k_{j}\right) D_{j}$.

- Users are interested in requesting content from the ISP cache if the cost of the content unit in the cache is less than the cost of the content unit in the CP.

\subsection{Utility Model}

Each ISP can cache the content or just forward the request to $\mathrm{CP}$ based on the 
utility that it gain. The utility function of each ISP is defined as the utility received by providing the network or content for users. Therefore, the utility function of each ISP is the difference between the total revenue and the fee:

$$
\begin{aligned}
& U_{j}=\left(p_{s_{j}}-p_{t}\right) D_{j}\left(\mathbf{p}_{s}, \mathbf{p}_{c}, \mathbf{q}_{s}, \mathbf{k}\right)\left(N-k_{j}\right)+\left(p_{s_{j}}+p_{c_{j}}-c_{j}\right) k_{j} D_{j}\left(\mathbf{p}_{s}, \mathbf{p}_{c}, \mathbf{q}_{s}, \mathbf{k}\right) \\
& -v_{j}\left(N-k_{j}\right) B_{j}
\end{aligned}
$$

Where $c_{j}$ the caching costs of unit of content. $c_{j} k_{j} D_{j}$ the fee of $I S P_{j}$ by serving the requested demand $k_{j} D_{j}$ from its cache. $\left(p_{s_{j}}+p_{c_{j}}-c_{j}\right) k_{j} D_{j}$ is the revenue of the $I S P_{j}$ by serving the requested demand $k_{j} D_{j}$ from its cache. The revenue of the $I S P_{j}$ is $\left(p_{s_{j}}-p_{t}\right) D_{j}\left(N-k_{j}\right)$ when the $I S P_{j}$ fulfils an amount of content request $D_{j}\left(N-k_{j}\right)$ by retrieving content from the CP. $v_{j}$ unit backhaul bandwidth cost. $B_{j}$ Backhaul bandwidth needed to serve the demand $D_{j} .\left(N-k_{j}\right) B_{j}$ the new backhaul bandwidth needed to serve the demand $\left(N-k_{j}\right) D_{j} . B_{j}$ is the backhaul bandwidth required by the ISP. It is increasing function w.r.t $D_{j}$ and QoS $q_{s_{j}}$ because a larger demand or higher QoS usually require a larger backhaul bandwidth. The quality of service $q_{s_{j}}$ can be defined by various metrics such as latency, jitter, or bandwidth. Latency is a measure of the delay that the traffic experiences as it traverses a network, and jitter is defined as the variation in that delay. Bandwidth is measured as the amount of data that can pass through a point in a network over time. Here, we define the QoS as the "expected delay". The expected delay is computed by the Kleinrock function that corresponds to the delay of M/M/1 queue with FIFO discipline or M/G/1 queue under processor sharing [2]. Similar to [2], instead of using the actual delay, we consider the reciprocal of its square root.

$$
q_{s_{j}}=\frac{1}{\sqrt{\text { Delay }}}=\sqrt{B_{j}\left(D_{j}, q_{s_{j}}\right)-D_{j}\left(\mathbf{p}_{s}, \mathbf{p}_{c}, \mathbf{q}_{s}, \mathbf{k}\right)}
$$

It means that:

$$
B_{j}=q_{s_{j}}^{2}+D_{j}\left(\mathbf{p}_{s}, \mathbf{p}_{c}, \mathbf{q}_{s}, \mathbf{k}\right)
$$

Then, the utility function of the $I S P_{j}$ given by the following formula:

$$
\begin{aligned}
& U_{j}=\left(p_{s_{j}}-p_{t}-v_{j}\right) D_{j}\left(\mathbf{p}_{s}, \mathbf{p}_{c}, \mathbf{q}_{s}, \mathbf{k}\right)\left(N-k_{j}\right)+\left(p_{s_{j}}+p_{c_{j}}-c_{j}\right) k_{j} D_{j}\left(\mathbf{p}_{s}, \mathbf{p}_{c}, \mathbf{q}_{s}, k\right) \\
& -v_{j}\left(N-k_{j}\right) q_{s_{j}}^{2}
\end{aligned}
$$

The deployment of the cache is beneficial for the ISP if and only if the revenue from cache $\left(p_{c_{j}}-c_{j} k_{j}\right) D_{j}$ are larger than backhaul bandwidth savings $v_{j} k_{j} B_{j}$.

\section{A Non-Cooperative Game Formulation}

For a precise formulation of a non-cooperative game, we have to specify (i) the number of players, (ii) the possible actions available to each player, and any constraints that may be imposed on them, (iii) the objective function of each player which she attempts to optimize. Here we will consider formulation of games where items (i)-(iii) above are relevant. 
Let $G=\left[\mathrm{l},\left\{P_{s_{j}}, Q_{s_{j}}, P_{c_{j}}, K_{j}\right\},\left\{U_{i}().\right\}\right]$ denote the non-cooperative price QoS price and number of cached items game (NPQPCG), where $I=\{1, \ldots, M\}$ is the index set identifying the ISPs. $P_{s_{j}}$ is the network access price strategy set of $I S P_{j}, Q_{s_{j}}$ is the QoS strategy set of $I S P_{j}, P_{c_{j}}$ is the content access price strategy set of $I S P_{j}$ and $k_{j}$ is the number of cached items strategy set of $I S P_{j} . U_{j}($.$) is the utility function of I S P_{j}$ defined in equation 9. We assume that the strategy spaces $P_{s_{j}}, Q_{s_{j}}, P_{c_{i}}$ and $K_{j}$ of each $I S P_{j}$ are compact and convex sets with maximum and minimum constraints. Thus, for each $I S P_{j}$ we consider as respective strategy spaces the closed intervals: $P_{s_{j}}=\left[\underline{p_{s_{j}}}, \overline{p_{s_{j}}}\right]$, $Q_{s_{j}}=\left[\underline{q_{s_{j}}}, \overline{q_{s_{j}}}\right], \quad P_{c_{j}}=\left[\underline{p_{c_{j}}}, \overline{p_{c_{j}}}\right] \quad$ and $\quad K_{j}=\left\lfloor\underline{k_{j}}, \overline{k_{j}}\right\rfloor . \quad$ Let the price vecto $\mathbf{p}_{s}=\left(p_{s_{1}}, \ldots, p_{s_{M}}\right)^{T} \in P_{s}=P_{s_{1}} \times P_{s_{2}} \times \ldots \times P_{s_{M}}, \quad$ QoS vector $\mathbf{q}_{s}=\left(q_{s_{1}}, \ldots, q_{s_{M}}\right)^{T} \in Q_{s}=Q_{s_{1}} \times Q_{s_{2}} \times \ldots \times Q_{s_{M}}, \quad$ price vector $p_{c}=\left(p_{c_{1}}, \ldots, p_{c_{M}}\right)^{T} \in P_{c}=P_{c_{1}} \times P_{c_{2}} \times \ldots \times P_{c_{M}}$. number of cached items vector $\mathbf{k}=\left(k_{1}, \ldots, k_{M}\right)^{T} \in K=K_{1} \times K_{2} \times \ldots \times K_{M}$ (where T represents the transpose operator).

In order to maximize their utilities, each $I S P_{j}$ decides a price $p_{s_{j}}$, QoS $q_{s_{j}}$, price $p_{c_{j}}$ and number of cached items $k_{j}$. Formally, the NPQCG problem can be expressed as:

$$
\max _{p_{s_{j}} \in P_{s_{j}}, q_{s_{j}} \in Q_{s_{j}}, p_{c_{j}} \in P_{c_{j}}, k_{j} \in K_{j}} U_{j}\left(\mathbf{p}_{s}, \mathbf{q}_{s}, \mathbf{p}_{c}, \mathbf{k}\right), \quad \forall j \in \mathrm{l} .
$$

\subsection{The Nash Equilibrium}

Nash Equilibrium (NE) is the most well-known solution to the non-cooperative games. Nash equilibrium is a fixed point of a non-cooperative game where no player can increase the value of its utility function through individual action.

We first will investigate the Nash equilibrium solution for the induced game as defined in the previous section. We will show that a Nash equilibrium solution exists and unique. We recall that a non-cooperative game $\mathrm{G}$ is called concave if all players' utility functions are strictly concave with respect to their corresponding strategies, [24].

According to, [24], a Nash equilibrium exists in a concave game if the joint strategy space is compact and convex, and the utility function that any given player seeks to maximize is concave in its own strategy and continuous at every point in the product strategy space. Formally, if the weighted sum of the utility functions with nonnegative weights:

$$
\psi=\sum_{j=1} x_{j} U_{j}, x_{j}>0 \forall j
$$

is diagonally strictly concave, this implies that the Nash equilibrium point is unique. Also, according to, [18] a Nash equilibrium is unique in a concave game, if the game satisfies the dominance solvability condition. 


\subsubsection{Joint Price $P_{s}$ Game}

A NPQPCG in network access price is defined for fixed $\mathbf{q}_{s} \in Q_{s}, \mathbf{p}_{c} \in P_{C}, \mathbf{k} \in K$ as $G\left(\mathbf{q}_{s}, \mathbf{p}_{c}, \mathbf{k}\right)=\left[\mathbf{l},\left\{P_{s_{j}}\right\},\left\{U_{j}\left(., \mathbf{q}_{s}, \mathbf{p}_{c}, \mathbf{k}\right)\right\}\right]$.

Defenition 1 A price vector $\mathbf{p}_{s}^{*}=\left(p_{s_{1}}^{*}, \ldots, p_{s_{M}}^{*}\right)$ is a Nash equilibrium of the NPQPCG $G\left(\mathbf{q}_{s}, \mathbf{p}_{c}, \mathbf{k}\right)$ if for every $j \in \mathrm{I}, U_{j}\left(p_{s_{j}}^{*}, \mathbf{p}_{s_{-j}}^{*}, \mathbf{q}_{s}, \mathbf{p}_{c}, \mathbf{k}\right) \geq U_{j}\left(p_{s_{j}}, \mathbf{p}_{s_{-j}}^{*}, \mathbf{q}_{s}, \mathbf{p}_{c}, \mathbf{k}\right)$ for all $p_{s_{j}} \in P_{s_{j}}$, where $\mathbf{p}_{-\mathbf{j}}=\left(p_{s_{1}}, \ldots, p_{s_{j-1}}, p_{s_{j+1}}, \ldots, p_{s_{M}}\right)$ is the M-1 dimensional vector of ISP price that does not contain price of $I S P_{j}$.

Theorem 1 For each $\mathbf{q}_{s} \in Q_{s}, \mathbf{p}_{c} \in P_{c}, \mathbf{k} \in K$, the game $\left[I,\left\{P_{s_{j}}\right\},\left\{U_{j}\left(., \mathbf{q}_{s}, \mathbf{p}_{c}, \mathbf{k}\right)\right\}\right.$ admits a unique Nash equilibrium.

Proof. To prove existence, we note that each ISPs strategy space $P_{s_{j}}$ is defined by all prices in the closed interval bounded by the minimum and maximum prices. Thus, the joint strategy space $P_{s}$ a nonempty, convex, and compact subset of the Euclidean space $R^{N}$. In addition, the utility functions are concave with respect to prices as can be seen from the second derivative test:

$$
\frac{\partial^{2} U_{j}}{\partial p_{s_{j}}^{2}}=-2 \alpha_{j}^{j} N \leq 0
$$

which ensures existence of a Nash equilibrium.

We use the following proposition that holds for a concave game [18]: If a concave game satisfies the dominance solvability condition:

$$
-\frac{\partial^{2} U_{j}}{\partial p_{s_{j}}^{2}} \geq \sum_{m, m \neq j}\left|\frac{\partial^{2} U_{j}}{\partial p_{s_{j}} \partial p_{s_{m}}}\right|
$$

then the game $\mathrm{G}$ admits a unique NE.

The mixed partial is written as:

$$
\frac{\partial^{2} U_{j}}{\partial p_{s_{j}} \partial p_{s_{m}}}=N \alpha_{j}^{m}
$$

Then,

$$
-\frac{\partial^{2} U_{j}}{\partial p_{s_{j}}^{2}}-\sum_{m, m \neq j}\left|\frac{\partial^{2} U_{j}}{\partial p_{s_{j}} \partial p_{s_{m}}}\right|=N\left(2 \alpha_{j}^{j}-\sum_{m, m \neq j} \alpha_{j}^{m}\right) \geq 0
$$

Thus, the Nash equilibrium point is unique.

\subsubsection{Joint Price $P_{c}$ Game}

A NPQPCG in network access price is defined for fixed $\mathbf{p}_{s} \in P_{s}, \mathbf{q}_{s} \in Q_{s}, \mathbf{k} \in K$ as $G\left(\mathbf{p}_{s}, \mathbf{q}_{s}, \mathbf{k}\right)=\left[\mathrm{l},\left\{P_{c_{j}}\right\},\left\{U_{j}\left(\mathbf{p}_{s}, \mathbf{q}_{s},,, \mathbf{k}\right)\right\}\right]$.

Defenition 2 A price vector $\mathbf{p}_{c}^{*}=\left(p_{c_{1}}^{*}, \ldots, p_{c_{M}}^{*}\right)$ is a Nash equilibrium of the NPQPCG 
$G\left(\mathbf{p}_{s}, \mathbf{q}_{s}, \mathbf{k}\right)$ if for every $j \in \mathrm{I}, U_{j}\left(\mathbf{p}_{s}, \mathbf{q}_{s}, p_{c_{j}}^{*}, \mathbf{p}_{c_{-j}}^{*}, \mathbf{k}\right) \geq U_{j}\left(\mathbf{p}_{s}, \mathbf{q}_{s}, p_{c_{j}}, \mathbf{p}_{c_{-j}}^{*}, \mathbf{k}\right)$ for all $p_{c_{j}} \in P_{c_{j}}$, where $\mathbf{p}_{c_{-j}}=\left(p_{c_{1}}, \ldots, p_{c_{j-1}}, p_{c_{j+1}}, \ldots, p_{c_{M}}\right)$.

Theorem 2 For each $\mathbf{p}_{s} \in P_{s}, \mathbf{q}_{s} \in Q_{s}, \mathbf{k} \in K$, the game $\left[\mathrm{I},\left\{P_{c_{j}}\right\},\left\{U_{j}\left(\mathbf{p}_{s}, \mathbf{q}_{s}, ., \mathbf{k}\right)\right\}\right]$ admits a unique Nash equilibrium.

Proof. To prove existence, we note that each ISPs strategy space $P_{c_{j}}$ is defined by all prices in the closed interval bounded by the minimum and maximum prices. Thus, the joint strategy space $P_{c}$ a nonempty, convex, and compact subset of the Euclidean space $R^{N}$. In addition, the utility functions are concave with respect to prices as can be seen from the second derivative test:

$$
\frac{\partial^{2} U_{j}}{\partial p_{c_{j}}^{2}}=-2 \gamma_{j}^{j} k_{j} \leq 0
$$

which ensures existence of a Nash equilibrium.

We use the following proposition that holds for a concave game [18]: If a concave game satisfies the dominance solvability condition:

$$
-\frac{\partial^{2} U_{j}}{\partial p_{c_{j}}^{2}} \geq \sum_{m, m \neq j}\left|\frac{\partial^{2} U_{j}}{\partial p_{c_{j}} \partial p_{c_{m}}}\right|
$$

then the game $\mathrm{G}$ admits a unique NE.

The mixed partial is written as:

$$
\frac{\partial^{2} U_{j}}{\partial p_{c_{j}} \partial p_{c_{m}}}=\gamma_{j}^{m} k_{j}
$$

Then,

$$
-\frac{\partial^{2} U_{j}}{\partial p_{c_{j}}^{2}}-\sum_{m, m \neq j}\left|\frac{\partial^{2} U_{j}}{\partial p_{c_{j}} \partial p_{c_{m}}}\right|=k_{j}\left(2 \gamma_{j}^{j}-\sum_{m, m \neq j} \gamma_{j}^{m}\right) \geq 0
$$

thus, the Nash equilibrium point is unique.

\subsubsection{Joint QoS Game}

A NPQPCG in QoS is defined for fixed $\mathbf{p}_{s} \in P_{s}, \quad \mathbf{p}_{c} \in P_{c}, \quad \mathbf{k} \in K$ as $G\left(\mathbf{p}_{s}, \mathbf{p}_{c}, \mathbf{k}\right)=\left[\mathrm{l},\left\{Q_{s_{j}}\right\},\left\{U_{j}\left(\mathbf{p}_{s}, ., \mathbf{p}_{c}, \mathbf{k}\right)\right\}\right]$.

defenition 3 A price vector $\mathbf{q}_{s}^{*}=\left(p_{s_{1}}^{*}, \ldots, p_{s_{M}}^{*}\right)$ is a Nash equilibrium of the NPQPCG $G\left(\mathbf{p}_{s}, \mathbf{p}_{c}, \mathbf{k}\right)$ if for every $j \in \mathrm{I}, U_{j}\left(\mathbf{p}_{s}, q_{s_{j}}^{*}, \mathbf{q}_{s_{-j}}^{*}, \mathbf{p}_{c}, \mathbf{k}\right) \geq U_{j}\left(\mathbf{p}_{s}, q_{s_{j}}, \mathbf{q}_{s_{-j}}^{*}, \mathbf{p}_{c}, \mathbf{k}\right)$ for all $q_{s_{j}} \in Q_{s_{j}}$, where $\mathbf{q}_{s_{-j}}=\left(q_{s_{1}}, \ldots, q_{s_{j-1}}, q_{s_{j+1}}, \ldots, q_{s_{M}}\right)$.

Theorem 3 For each $\mathbf{p}_{s} \in P_{s}, \mathbf{p}_{c} \in P_{c}, \mathbf{k} \in K$, the game $\left[\mathrm{I},\left\{Q_{s_{j}}\right\},\left\{U_{j}\left(\mathbf{p}_{s}, ., \mathbf{p}_{c}, \mathbf{k}\right)\right\}\right]$ admits a unique Nash equilibrium.

Proof. To prove existence, we note that each ISPs strategy space $Q_{s_{j}}$ is defined by all QoSs in the closed interval bounded by the minimum and maximum QoSs. Thus, the joint 
strategy space $Q_{s}$ a nonempty, convex, and compact subset of the Euclidean space $R^{M}$. In addition, the utility functions are concave with respect to QoSs as can be seen from the second derivative test:

$$
\frac{\partial^{2} U_{j}}{\partial q_{s_{j}}^{2}}=-2 v_{j}\left(N-k_{j}\right) \leq 0
$$

which ensures existence of a Nash equilibrium.

In order to prove uniqueness, we follow, [24], and define the weighted sum of user utility functions.

$$
\psi\left(\mathbf{q}_{s}, \mathbf{x}\right)=\sum_{j=1}^{M} x_{j} U_{j}\left(q_{s_{j}}, \mathbf{q}_{s_{-j}}\right)
$$

The pseudo-gradient of 19 is given by:

$$
v\left(\mathbf{q}_{s}, x\right)=\left\lfloor x_{1} \nabla U_{1}\left(q_{s_{1}}, \mathbf{q}_{s_{-1}}\right), \ldots, x_{M} \nabla U_{M}\left(q_{s_{M}}, \mathbf{q}_{s_{-M}}\right)\right\rfloor^{T}
$$

The Jacobian matrix $\mathbf{J}$ of the pseudo-gradient (w.r.t.q) is written:

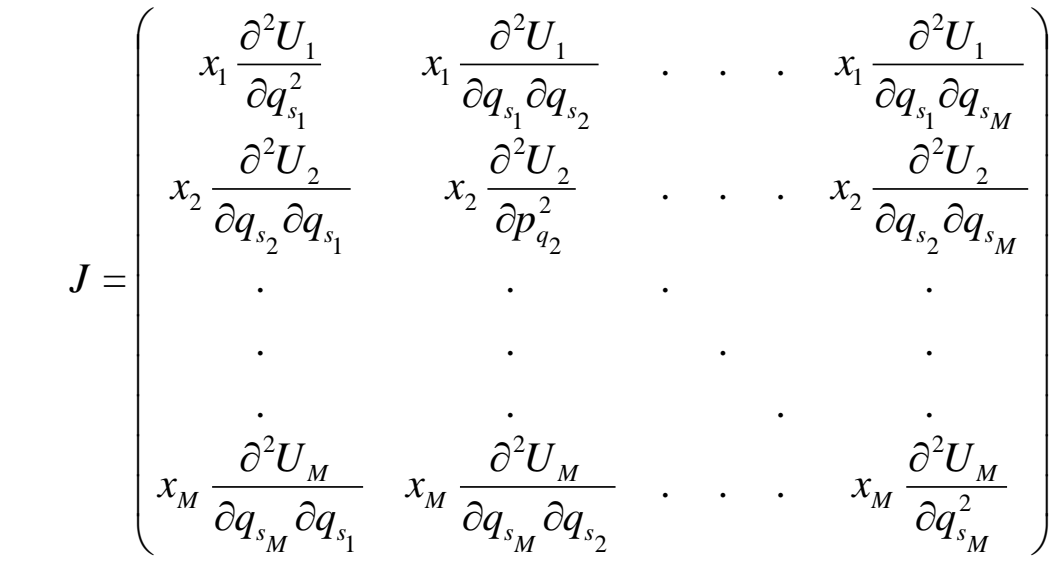

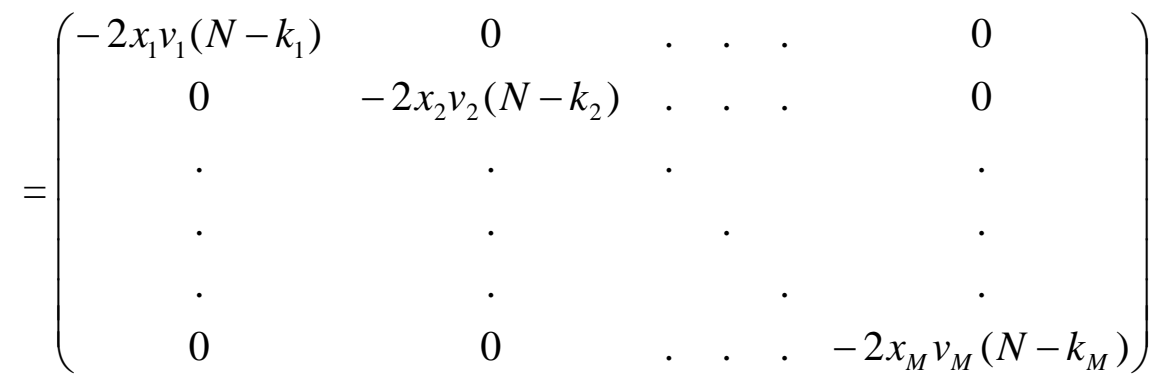

Thus, $\mathbf{J}$ is a diagonal matrix with negative diagonal elements. This implies that $\mathbf{J}$ is negative definite. Henceforth $\left[J+J^{T}\right]$ is also negative definite, and according to Theorem (6) in, [24], the weighted sum of the utility functions $\psi\left(q_{s}, x\right)$ is diagonally strictly concave. Thus, the Nash equilibrium point is unique.

\subsubsection{Joint Cache Game}

A NPQPCG in number of cached content is defined for fixed $\mathbf{p}_{s} \in P_{s}, \mathbf{q}_{s} \in Q_{s}$, $\mathbf{p}_{c} \in P_{c}$ as $G\left(\mathbf{p}_{s}, \mathbf{q}_{s}, \mathbf{p}_{c}\right)=\left[\mathrm{l},\left\{K_{j}\right\},\left\{U_{j}\left(\mathbf{p}_{s}, \mathbf{q}_{s}, \mathbf{p}_{\mathbf{c}},.\right\}\right]\right.$. 
Defenition 4 A price vector $\mathbf{k}^{*}=\left(k_{1}^{*}, \ldots, k_{M}^{*}\right)$ is a Nash equilibrium of the NPQPCG $G\left(\mathbf{p}_{s}, \mathbf{q}_{s}, \mathbf{p}_{c}\right)$ if for every $j \in \mathrm{I}, U_{j}\left(\mathbf{p}_{s}, \mathbf{q}_{s}, \mathbf{p}_{c}, k_{j}^{*}, \mathbf{k}_{-j}^{*}\right) \geq U_{j}\left(\mathbf{p}_{s}, \mathbf{q}_{s}, \mathbf{p}_{c}, k_{j}^{*}, \mathbf{k}_{-j}^{*}\right)$ for all $k_{j} \in K_{j}$, where $\mathbf{k}_{-j}=\left(k_{1}, \ldots, k_{j-1}, k_{j+1}, \ldots, k_{M}\right)$.

Theorem 4 For each $\mathbf{p}_{s} \in P_{s}, \mathbf{q}_{s} \in Q_{s}, \mathbf{p}_{c} \in P_{c}$ the game $\left[\mathrm{I},\left\{K_{j}\right\},\left\{U_{j}\left(\mathbf{p}_{s}, \mathbf{q}_{s}, \mathbf{p}_{\mathbf{c}},.\right\}\right]\right.$ admits a unique Nash equilibrium.

Proof: To prove existence, we note that each ISPs strategy space $K_{j}$ is defined by all number of cached items in the closed interval bounded by the minimum and maximum number of cached items. Thus, the joint strategy space $\mathrm{K}$ a nonempty, convex, and compact subset of the Euclidean space $R^{M}$. In addition, the utility functions are concave with respect to $\mathrm{k}$ as can be seen from the second derivative test:

$$
\frac{\partial^{2} U_{j}}{\partial k_{j}^{2}}=-2 \sigma_{j}^{j}\left(c_{j}-p_{t}-v_{j}-p_{c_{j}}\right)
$$

If $c_{j}>p_{t}+v_{j}+p_{c_{j}}$ then

$$
\frac{\partial^{2} U_{j}}{\partial k_{j}^{2}} \leq 0
$$

which ensures existence of a Nash equilibrium.

We use the following proposition that holds for a concave game [18]: If a concave game satisfies the dominance solvability condition:

$$
-\frac{\partial^{2} U_{j}}{\partial k_{j}^{2}} \geq \sum_{m, m \neq j}\left|\frac{\partial^{2} U_{j}}{\partial k_{j} \partial k_{m}}\right|
$$

then the game admits a unique NE.

The mixed partial is written as:

$$
\frac{\partial^{2} U_{j}}{\partial k_{j} \partial k_{m}}=\left(c_{j}-p_{t}-v_{j}-p_{c_{j}}\right) \sigma_{j}^{m}
$$

Also,

$$
-\frac{\partial^{2} U_{j}}{\partial k_{j}^{2}}-\sum_{m, m \neq j}\left|\frac{\partial^{2} U_{j}}{\partial k_{j} \partial k_{m}}\right|=\left(c_{j}-p_{t}-v_{j}-p_{c_{j}}\right)\left(2 \sigma_{j}^{j}-\sum_{m, m \neq j} \sigma_{j}^{m}\right)
$$

Thus,

$$
-\frac{\partial^{2} U_{j}}{\partial k_{j}^{2}}-\sum_{m, m \neq j}\left|\frac{\partial^{2} U_{j}}{\partial k_{j} \partial k_{m}}\right| \geq 0
$$

Then, the Nash equilibrium point is unique.

\subsubsection{Learning Nash Equilibrium}

The section mentioned above shows clearly that the Nash equilibrium is unique. Now, we turn to develop a fully algorithms that converge quickly to Nash equilibrium. However, we assume that each ISP has a perfect information on strategies of its competitors. Each ISP fixes its desirable strategies in order to maximize its own profit. Then, each ISP can observe the policy taken by its competitors in previous rounds and input them in its decision process to update its policy. Therefore, the best response algorithm will converge a to unique equilibrium. The best response algorithm is summarized in algorithm 1 . 


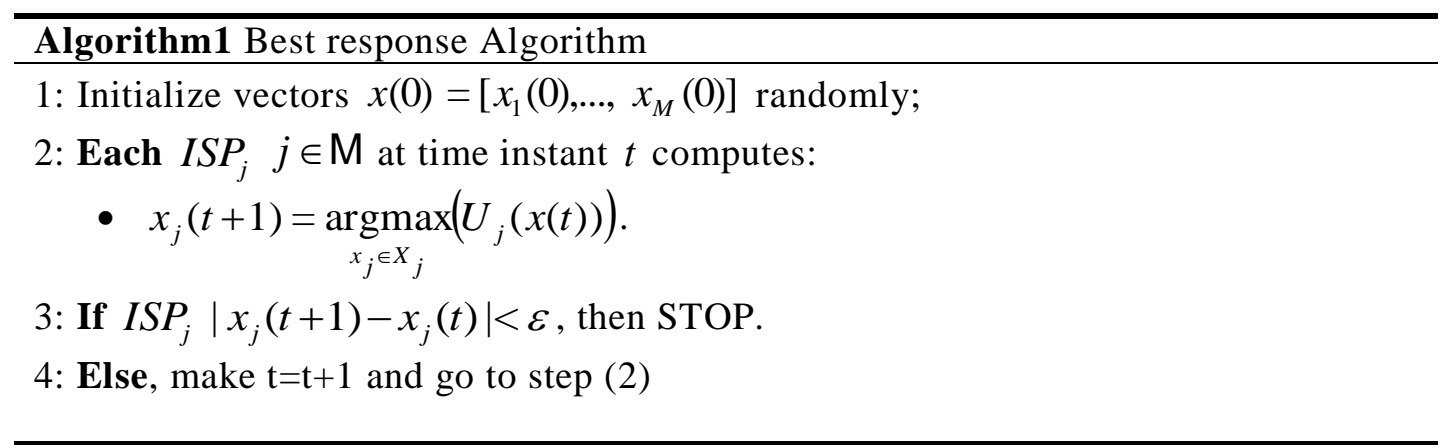

Such as:

- $\mathrm{x}$ denotes the vector $p_{s}$, vector $q_{s}$, vector $p_{c}$ or vector $k$.

- $X_{j}$ denotes the policy profile price $P_{s_{j}}$, policy profile QoS $Q_{s_{j}}$, policy profile price $P_{c_{j}}$ or policy profile of number of cached items $K_{j}$.

\subsubsection{Price of Anarchy}

Closely linked to the notion of efficiency or global performance is the concept of the price of anarchy, a term coined by Koutsoupias and Papadimitriou [15]. That metric quantifies the loss of efficiency due to the selfishness of the actors involved, by comparing the outcome of the non-cooperative setting (the Nash equilibria) with the most efficient reachable outcome (that we call the social optimum).

The price of anarchy (PoA) is defined as the ratio between the performance measures of the worst equilibrium and the optimal outcome. A PoA close to 1 indicates that the equilibrium is approximately socially optimal, and thus the consequences of selfish behavior are relatively benign.

In [7] we measure the loss of efficiency due to actors' selfishness as the quotient between the social welfare obtained at the Nash equilibrium and the maximum value of the social welfare:

$$
P o A=\frac{\min _{p_{s}, p_{c}, q_{s}, k} W_{N E}\left(p_{s}, p_{c}, q_{s}, k\right)}{\max _{p_{s}, p_{c}, q_{s}, k} W\left(p_{s}, p_{c}, q_{s}, k\right)}
$$

Where $W\left(p_{s}, p_{c}, q_{s}, k\right)=\sum_{j=1}^{M} U_{i}\left(p_{s}, p_{c}, q_{s}, k\right)$ a social welfare function and $W_{N E}\left(p_{s}^{*}, p_{c}^{*} q_{s}^{*}, k^{*}\right)=\sum_{j=1}^{M} U_{i}\left(p_{s}, p_{c}, q_{s}, k\right)$ is a sum of utilities of all actors at Nash Equilibrium.

\section{Numerical Investigations}

So far, we have completed all theoretical analyses and in this section, we propose to numerically study the gaming market taking account of previous expression of utility of the ISPs. For illustrative purposes, we consider two homogeneous ISPs seeking to maximize their earnings. 


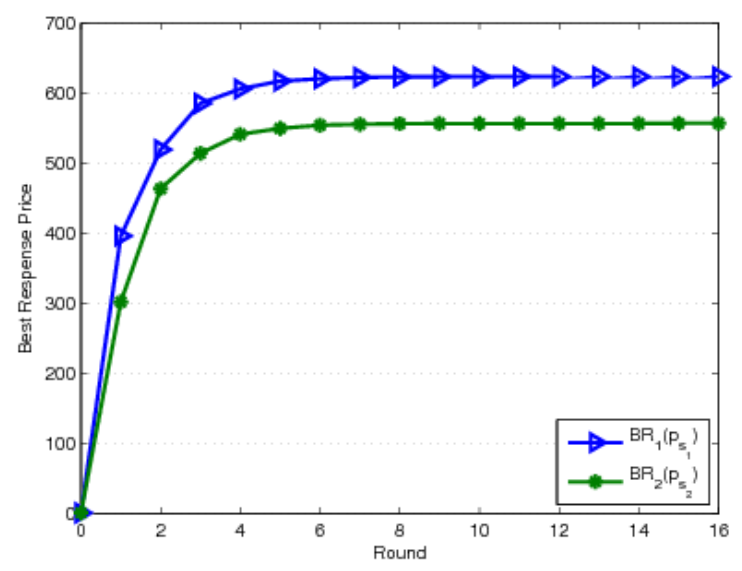

Figure 2. Network Access Price Game: Convergence to the Network Access Price Nash Equilibrium

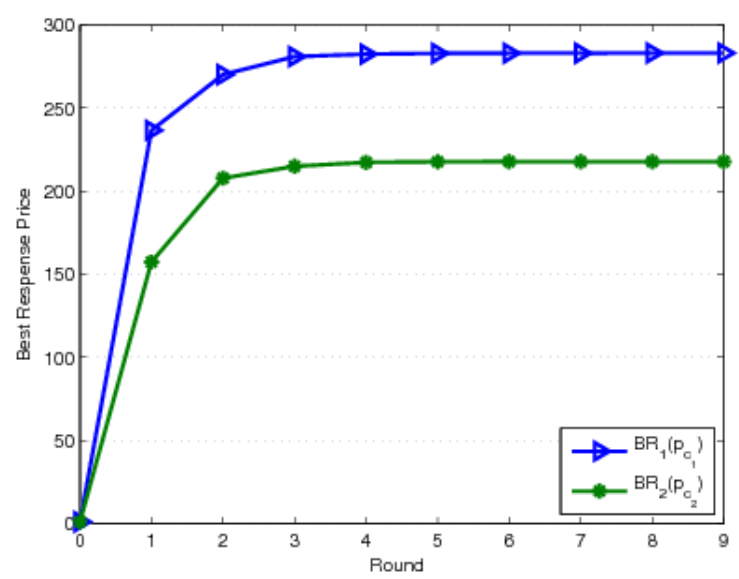

Figure 3. Price to Access to the Content in Cache Game: Convergence to the Price to Access to the Content in Cache Nash Equilibrium

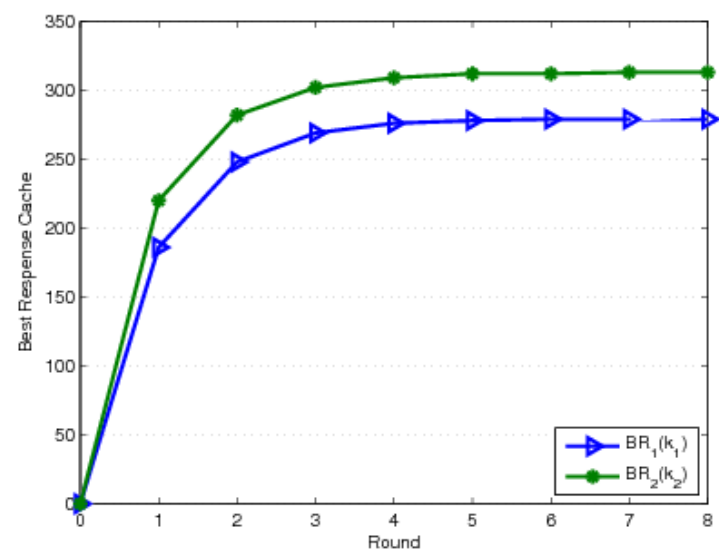

Figure 4. Number Cached Items Game: Convergence to the Number of Cached Items at Nash Equilibrium 


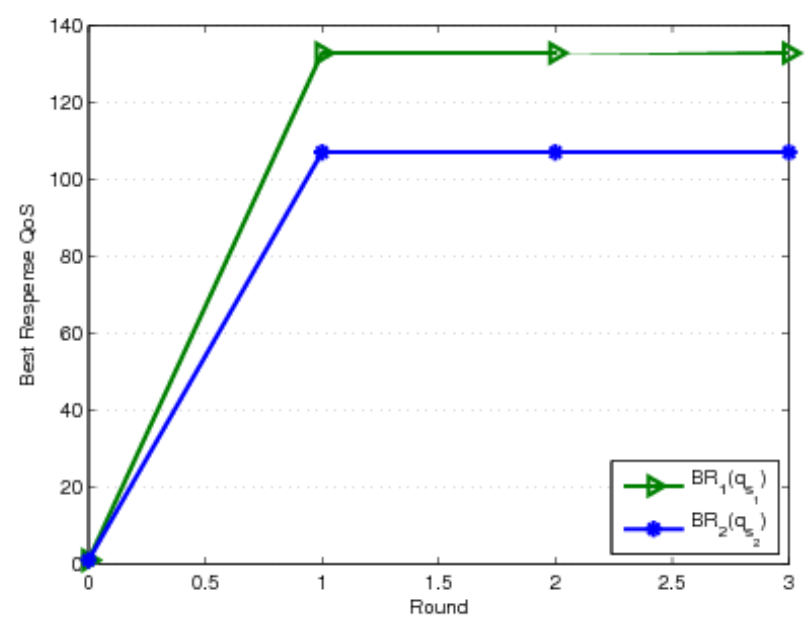

Figure 5. QoS game: Convergence to the QoS Nash Equilibrium

The Figures 2, 3, 4 and 5 present respectively curves of the convergence to Nash equilibrium price, QoS and number of cached items. It is clear that the best response algorithm converges to the unique Nash equilibrium price, QoS and number of cached items. We also remark that the speed of convergence is relatively high, so in this simulation Algorithm 1 is capable of efficiently converging to Nash equilibrium price, number of cached item and QoS.

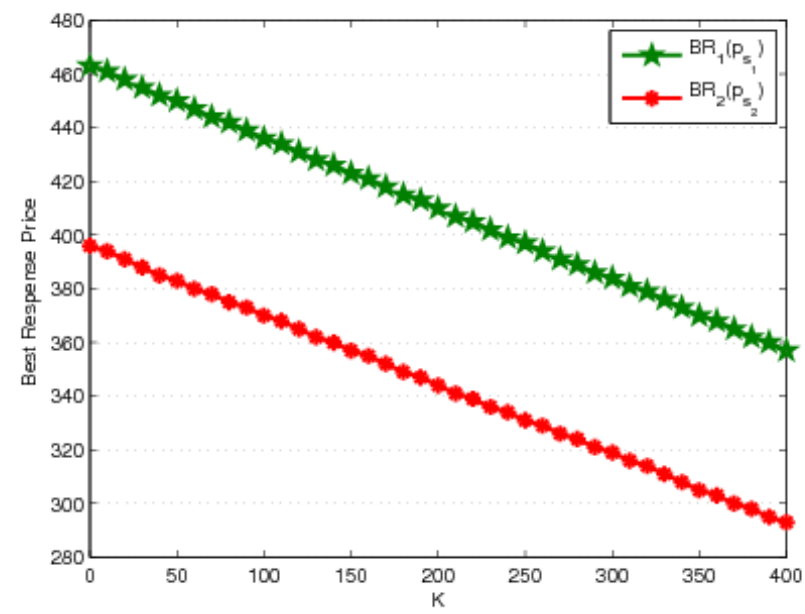

Figure 6. Equilibrium Network Access Price as a Function of Number's Cached Items

In Figure 6 we plot network access price $p_{s}$ as a function of number's cached items $\mathrm{k}$. price $p_{s}$ decreases with respect to number of cached items $\mathrm{k}$. When $\mathrm{k}$ increases, the fraction of content requests satisfied from the ISP cache increases. Then, the transmission fee and cost of of bandwidth decreases. Thus, the revenue of ISPs increases. Therefore, the ISP decreases its access price in order to induce increased demand from the users (see relationship between price $p_{s}$ and demand in equation 1). 


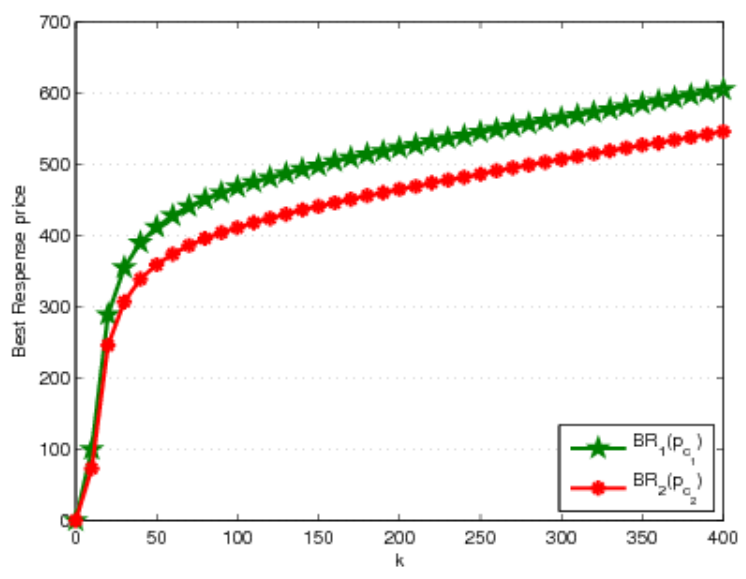

Figure 7. Equilibrium Prices as a Function of Number's Cached Items

Figure 7 shows the price to access to the content in the cache as function of the number of cached items $\mathrm{k}$. From the figure, we note that $p_{c}$ increase with respect to $\mathrm{k}$. When $\mathrm{k}$ increase, the cost of caching increase. Thus, the ISP needs to slightly increase its price $p_{c}$ to compensate the increase in the caching cost. However, the ISPs must control the price $p_{c}$, to not exceed the content access price of $\mathrm{CP}$, in order to motivate users to request more content from the ISP cache.

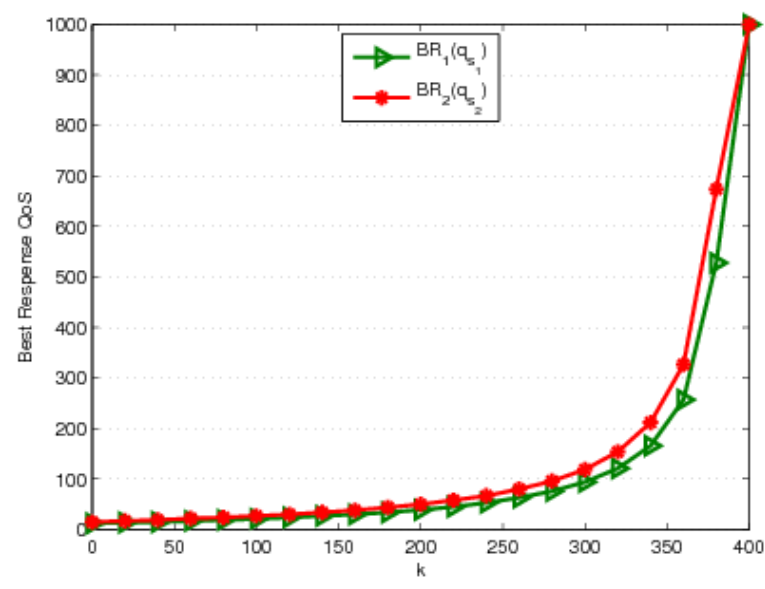

Figure 8. Equilibrium QoS as a Function of Number's Cached Items

Figure 8 represent the impact of $\mathrm{k}$ on quality of service QoS $q_{s}$. QoS $q_{s}$ increase with respect to $\mathrm{k}$. When $\mathrm{k}$ increase, the fraction of content requests satisfied from the ISP cache increases. Then, the bandwidth required satisfying content requests form CP decreases. In fact, the cost of bandwidth decreases. Therefore, ISPs invests for more bandwidth, so as to offer better QoS in order to induce increased demand from the users (see the relationship between QoS and demand in equation 1). Caching gives the ISPs more freedom to improve QoS. 


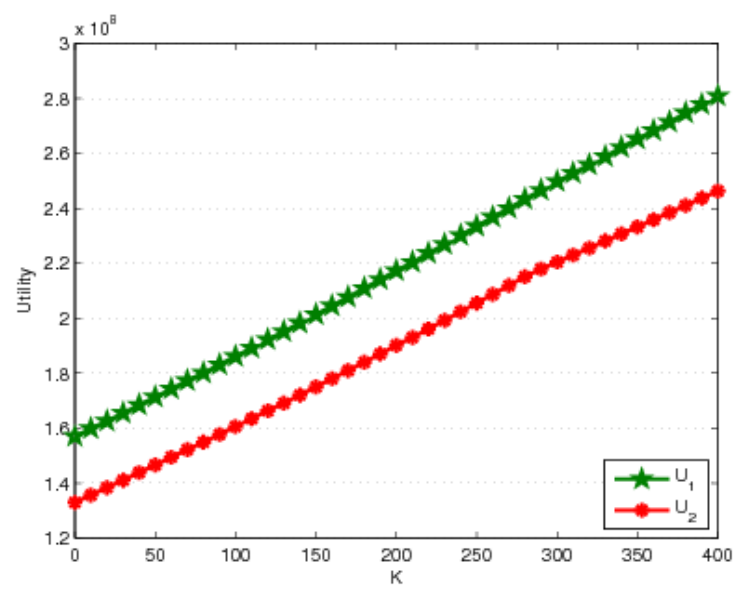

Figure 9. Utility as a Function of Number's Cached Items

We plot the expected utility of ISPs as a function of number's cached items $\mathrm{k}$ in Figure 9. Utility increase with respect to the number of cached items $\mathrm{k}$. When $\mathrm{k}$ increases, the transmission fee decrease, the cost of bandwidth decrease and demand increase. In fact, the revenue of ISPs increases. Caching increases ISPs revenue.

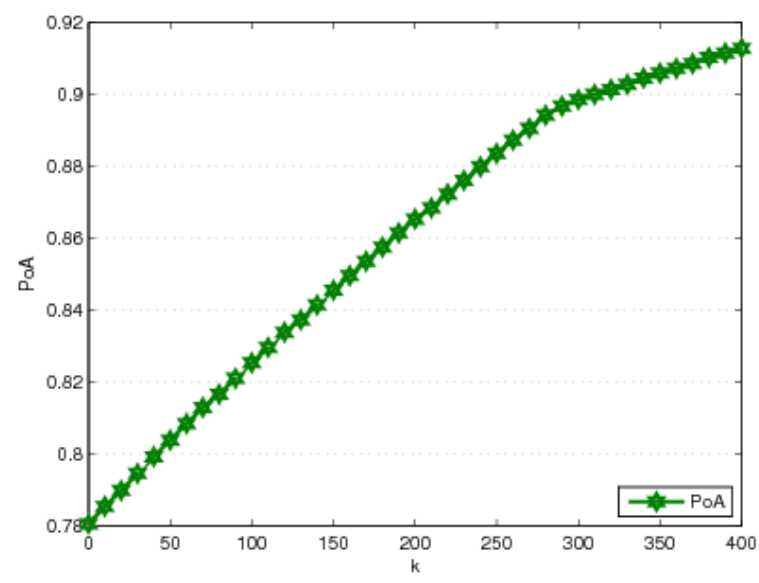

Figure 10. Price of Anarchy as a Function of Number's Cached Items

Figure 10 shows the PoA variation curve as a function of numbers of cached items $\mathrm{k}$. In that figure, we first notice that the price of anarchy increases with respect to $\mathrm{k}$. When $\mathrm{k}$ is lower, the price of anarchy is low and what shows that the sum of the optimal utilities is greater than the sum of utilities to equilibrium; which shows that the ISPs are selfish and each one seeks to maximize its profit. However, when $\mathrm{k}$ increases the price of anarchy approaches 1, the ISPs are not selfish and each one takes into consideration the strategy of his opponent to finally fall into the state of equilibrium. As a result, caching is beneficial for ISPs.

\section{Conclusion}

In this paper, we study competition between ISP in ICN using mathematical tools game theory. We modeled competition between ISP as a function of four-parameter network access price, quality of service, price to access to the content in the cache and a number of items in the cache. We proved the existence and uniqueness of Nash equilibrium in a competitive ICN market under our proposed model. Next, we 
describe a learning mechanism that allows each ISPs to discover accurately and rapidly its equilibrium policies. In addition, we use the price of anarchy to evaluate the effectiveness of Nash equilibrium. Our work also contributes to a better understanding the impact of caching investments on pricing, QoS and the utility of the ISPs under the proposed model. The results show that ISPs and users can benefit from caching investments.

As future work, we plan to add content popularity to our analysis. Finally, we have analyze caching, QoS and pricing in a non-cooperative context in ICNs where ISPs compete for maximizing their profit. It will be valuable to study also a cooperative context where ISPs form coalitions for sharing profits. Modeling customer behavior by using the Luce probabilistic model that incorporates bounded rational choice of customers [25].

\section{References}

[1] B. Ahlgren, C. Dannewitz, C. Imbrenda, D. Kutscher and B. Ohlman, "A survey of information-centric networking", IEEE Communications Magazine, vol. 50, no. 7, (2012).

[2] R. El Azouzi, E. Altman and L. Wynter, "Telecommunications network equilibrium with price and quality-of-service characteristics", Edited J. Charzinski, R. Lehnert and P. Tran-Gia, Providing Quality of Service in Heterogeneous Environments Proceedings of the 18th International Teletraffic Congress ITC-18 in Teletraffic Science and Engineering, Elsevier, (2003), pp. 369-378.

[3] F. Bernstein and A. Federgruen, "A general equilibrium model for industries with price and service competition", Operations research, vol. 52, no. 6, (2004), pp. 868-886.

[4] M. Chen, W. Saad, C. Yin and M. Debbah, "Echo State Networks for Proactive Caching in Cloud-Based Radio Access Networks With Mobile Users", IEEE Transactions on Wireless Communications, vol. 16, no. 6, (2017), pp. 3520-3535.

[5] M. Chen, W. Saad, C. Yin and M. Debbah, "Echo state networks for proactive caching and content prediction in cloud radio access networks", Globecom Workshops (GC Wkshps), 2016 IEEE, (2016), pp. 1-6.

[6] V. Douros, S. Elayoubi, E. Altman and Y. Hayel, "Caching Games between Content Providers and Internet Service Providers", Performance Evaluation, vol. 113, (2017), pp. 13-25.

[7] L. Guijarro, V. Pla, J. R. Vidal and J. Martinez-Bauset, "Analysis of price competition under peering and transit agreements in Internet Service provision to peer-to-peer users", 2011 IEEE Consumer Communications and Networking Conference (CCNC), (2011), pp. 1145-1149.

[8] M. Hajimirsadeghi, N. B. Mandayam and A. Reznik, "Joint caching and pricing strategies for information centric networks", Global Communications Conference (GLOBECOM), 2015 IEEE, (2015), pp. 1-6.

[9] K. Hamidouche, W. Saad and M. Debbah, "Many-to-many matching games for proactive social-caching in wireless small cell networks", Modeling and Optimization in Mobile, Ad Hoc, and Wireless Networks (WiOpt), 2014 12th International Symposium, (2014), pp. 569-574.

[10] K. Hamidouche, W. Saad, M. Debbah and H.V. Poor, "Mean-field games for distributed caching in ultra-dense small cell networks", American Control Conference (ACC), (2016), pp. 4699-4704.

[11] V. Jacobson, D. K. Smetters, J. D. Thornton, M. F. Plass, N. H. Briggs and R. L. Braynard, "Networking named content", Proceedings of the 5th international conference on Emerging networking experiments and technologies, ACM, (2009), pp. 1-12.

[12] H. Jin, D. Xu, C. Zhao and D. Liang, "Information-centric mobile caching network frameworks and caching optimization: a survey", EURASIP Journal on Wireless Communications and Networking, vol. 2017, no. 1, (2017).

[13] F. Kocak, G. Kesidis, T.-M. Pham and S. Fdida, "The Effect of Caching on a Model of Content and Access Provider Revenues in Information-centric Networks", IEEE, (2013), pp. 45-50.

[14] T. Koponen, M. Chawla, B. Chun, A. Ermolinskiy, K. H. Kim, S. Shenker and I. Stoica, "A dataoriented (and beyond) network architecture", ACM SIGCOMM Computer Communication Review, ACM, (2007), pp. 181-192.

[15] E. Koutsoupias and C. Papadimitriou, "Worst-case equilibria", Annual Symposium on Theoretical Aspects of Computer Science, Springer, (1999), pp. 404-413.

[16] J. Knsemller, N. Zhang, K. Berg and J. Soares, "A game-theoretic evaluation of an ISP business model in caching", Information Systems Frontiers, (2016).

[17] J. Knsemllern, N. Zhang and J. Soares, "ISP business models in caching”, Data Engineering Workshops (ICDEW), 2013 IEEE 29th International Conference on IEEE, (2013), pp. 279-285.

[18] S. Lasaulce, M. Debbah and E. Altman, "Methodologies for analyzing equilibria in wireless games", IEEE Signal Processing Magazine, vol. 26, no. 5, (2009), pp. 41-52. 
[19] I. Pappalardo, G. Quer, B. D. Rao and M. Zorzi, "Caching strategies in heterogeneous networks with D2D, small BS and macro BS communications", 2016 IEEE International Conference on Communications (ICC), (2016), pp. 1-6.

[20] T.-M. Pham, "Analysis of isp caching in information-centric networks", Computing \& Communication Technologies-Research, Innovation, and Vision for the Future (RIVF), 2015 IEEE RIVF International Conference on IEEE, (2015), pp 151-156.

[21] T.-M. Pham, S. Fdida and P. Antoniadis, "Pricing in information-centric network interconnection", IFIP Networking Conference, IEEE, (2013), pp. 1-9.

[22] T.-M. Pham, M. Minoux, S. Fdida and M. Pilarski, "Optimization of content caching in content-centric network", (2017).

[23] K. Poularakis and L. Tassiulas, "Exploiting user mobility for wireless content delivery", Information Theory Proceedings (ISIT), 2013 IEEE International Symposium on IEEE, (2013), pp. 1017-1021.

[24] J. B Rosen, "Existence and Uniqueness of Equilibrium Points for Concave N-Person Games", Econometrica, vol. 33, no. 3, (1965), pp. 520.

[25] D. Ait Omar, M. Outanoute, M. Baslam, M. Fakir and B. Bouikhalne, "Joint Price and QoS Competition with Bounded Rational Customers", Networked Systems: 5th International Conference, NETYS 2017, Marrakech, Morocco, Proceedings, A. El Abbadi and B. Garbinato, Eds., Cham, (2017) May 17-19, pp. 457-471. 